\title{
Dominant modes in hydromagnetic stability of channel flow with porous walls
}

\author{
M. LAMINE ${ }^{1}$, A. HIFDI ${ }^{1}$, \\ 1. Laboratory of Mechanics, Faculty of Sciences Aïn-Chock,University Hassan II , Casablanca, Morocco, \\ mustapha.lamine@gmail.com
}

\begin{abstract}
:
A linear stability analysis of a plane channel flow with porous walls under a uniform cross-flow and an external transverse magnetic field is explored. The physical problem is governed by a system of combined equations of the hydrodynamic and those of Maxwell. The perturbed problem of base state leads to a modified classical OrrSommerfeld equation which is solved numerically using the Chebyshev spectral collocation method. The combined effects of the cross-flow Reynolds number and the Hartmann number on the dangerous mode of hydromagnetic stability are investigated.The study shows that, the magnetic field tends to suppress the instability occurred by cross-flow. This stabilizing effect becomes perceptible when the magnetic field produces a mode transition from walls mode to that of the center.
\end{abstract}

Keywords: Dominant modes, cross-flow, magnetic field, porous walls, hydromagnetic stability, Spectral method.

\section{Introduction}

Stability analysis of flows between two porous plates has been considered as an active research area since its deep technological importance in several industrial applications: the biomedical industry, paper making, filtration systems, environmental engineering and aeronautics. Several processes and investigations have been performed for controlling the stability of this flow's type, e.g., crossflow, magnetic field, pressure oscillation. This configuration was initially carried out theoretically by Berman [1] in which a description of the laminar flow is discussed. In addition, Hains [2] and Sheppard [3] performed a linear stability analysis of the channel flow. In these studies, the authors have shown that a modest amount of uniform injection/suction of the same fluid produced a significant increase in critical Reynolds number. Indeed, they have defined two Reynolds numbers related respectively to, the maximum symmetric plane Poiseuille velocity and the cross-flow velocity. An extension of previous works [2,3], Fransson and Alfredsson [4] have made corrections to the problems discussed in $[2,3]$. In particular, they separated the effects of the velocity distribution from those of the magnitude of the velocity in the basic state, by using the maximal channel velocity of plane Poiseuille flow with the presence of a cross-flow as their characteristic velocity. Recently, Lamine and Hifdi [5] showed that the sense of the cross-flow has no effect on the stability of Poiseuille flow.

In this paper, we extend the previous work [4] for an electrically conducting fluid to include the effect of a uniform external transverse magnetic field. In this way, several investigations have been devoted to illustrate the effect of external magnetic field on the hydrodynamic stability of the channel. For instance, the governing equations of the flow's stability can be simplified when the considered fluid is highly electrically conducting [6], i.e., the magnetic Reynolds number is very small. In[6], it has been found that, by using an asymptotic method, the magnetic field has a stabilizing effect. The validity of Lock's assumption [6] has been checked by Takashima [7] and it was show that, except for the case where the magnetic Prandtl number is sufficiently small, the magnetic field has a stabilizing effect.

The combined effects of an imposed external magnetic field and uniform cross-flow have been examined in several investigations. For instance. Mehta and Jain [8] have determined analytically an exact basic solution for laminar flow in a rectangular channel with porous walls under magnetic field.

The physical motivation of our study to assess how the combined effects of an external transverse magnetic field and cross-flow (injection / suction) on the hydrodynamic stability of the dangerous mode in parallel shear flow and also for determining the nature of the dominant eigenmodes. This paper is organized as follows. The studied configuration and the steady basic flow solution are defined in section 2. Section 3 is devoted to performing a linear stability analysis and to present the numerical method used to solve the stability problem. In section 4 , pertinent results are discussed quantitatively.

\section{Physical model and mathematical formu-}

\section{lation}

Considering a highly electrically conducting viscous incompressible fluid in a plane channel formed by two infinite porous parallel plates separated by a fixed distance $2 h$. A cross-flow of constant velocity, $v_{o}$, is applied to the fluid in the transverse direction with the presence of a uni- 
form external magnetic field, $B_{o}$, parallel to the $y^{*}$-axis. The injection takes place in the upper plate, $y^{*}=+h$, while the suction is at the lower plate, $y^{*}=-h$. The mathematical equations modelling the flow in their dimensional forms $(*)$ are

$$
\begin{aligned}
\operatorname{Div} \mathbf{V}^{*}=0 \quad & \quad \operatorname{Div}^{*}=0 \\
\rho\left[\frac{\partial \mathbf{V}^{*}}{\partial t^{*}}+\left(\mathbf{V}^{*} \nabla\right) \cdot \mathbf{V}^{*}\right]= & -\nabla P^{*}+\rho \nu \Delta \mathbf{V}^{*} \\
& +\frac{1}{\rho \mu}\left[\left(\nabla \wedge \mathbf{B}^{*}\right) \wedge \mathbf{B}^{*}\right] \\
\frac{\partial \mathbf{B}^{*}}{\partial t^{*}}-\nabla \wedge\left(\mathbf{V}^{*} \wedge \mathbf{B}^{*}\right) & =\frac{1}{\sigma \mu} \Delta \mathbf{B}^{*}
\end{aligned}
$$

where $\rho, \nu, \mu, \sigma$ and $P^{*}$ and $\mathbf{V}^{*}$ are, respectively, the fluid density, the kinematic viscosity, the magnetic permeability, is the electrical conductivity, the pressure and the velocity vector. Further, $\mathbf{B}^{*}$ is the magnetic field strength and $t^{*}$ is the time. The boundary conditions at the walls $\left(y^{*}= \pm h\right)$ are

$$
V_{x^{*}}^{*}=0 ; V_{y^{*}}^{*}=v_{0} ; B_{x^{*}}^{*}=0 ; B_{y^{*}}^{*}=B_{0}
$$

Using reference variables $h, U_{\max }, h U_{\max }^{-1}, \rho U_{\max }^{2}$ and $B_{o}$ for, respectively, length, velocity, time, pressure and magnetic field strength $\left(U_{\max }\right.$ represents the maximum streamwise velocity), the base-state solutions in nondimensional forms can be written as

$$
\begin{aligned}
& V_{b_{x}}(y)=\frac{\sinh \left(a_{1}\right)\left[e^{a_{2} y}-e^{a_{2} Y}\right]-\sinh \left(a_{2}\right)\left[e^{a_{1} y}-e^{a_{1} Y}\right]}{\sinh \left(a_{1}\right)\left[e^{a_{2} Y}-\cosh \left(a_{2}\right)\right]-\sinh \left(a_{2}\right)\left[e^{a_{1} Y}-\cosh \left(a_{1}\right)\right]}+1 \\
& B_{b_{x}}(y)=\frac{R_{m}}{a_{1} a_{2}}\left[\frac{\sinh \left(a_{1}\right)\left[e^{a_{2} y}-e^{a_{2}}\right] a_{1}-\sinh \left(a_{2}\right)\left[e^{a_{1} y}-e^{a_{1}}\right] a_{2}}{\sinh \left(a_{1}\right)\left[e^{a_{2} Y}-\cosh \left(a_{2}\right)\right]-\sinh \left(a_{2}\right)\left[e^{a_{1} Y}-\cosh \left(a_{1}\right)\right]}\right]
\end{aligned}
$$

with

$Y=\frac{1}{a_{2}-a_{1}} \log \left[\frac{a_{1}}{a_{2}} \frac{\sinh \left(a_{2}\right)}{\sinh \left(a_{1}\right)}\right] ; a_{1,2}=\frac{1}{2}\left[-R_{C} \pm \sqrt{R_{C}^{2}+4 M^{2}}\right]$

These basic solutions depend on the cross-flow Reynolds number, $R_{C}$, the Hartmann number, $M$, and for basic magnetic induction depends also on the magnetic Reynolds number, $R_{m}$. These parameters are defined, respectively by,

$$
R_{C}=\frac{v_{o} h}{\nu} ; M=B_{o} h \sqrt{\frac{\sigma}{\rho \nu}} ; \quad R_{m}=\frac{U_{\max } h}{\lambda}
$$

It can be verified that, in the limit $M \longrightarrow 0$ with $R_{C}=0$, the classical parabolic profile of plane Poiseuille flow (PPF), $\left(1-y^{2}\right)$, is retrieved. Also, for $R_{C}=0$, the basic velocity (Eq.5) reduces to that found by Takashima [7], corresponding to PPF under an external transverse magnetic field and became to that give by Fransson and Alfredsson [4] when $M \longrightarrow 0$ and $R_{C} \neq 0$.

\section{Linear stability analysis}

Considering the linear stability of the non-dimensional form of governing equations (1)-(3) with its boundary conditions, Eq.(4), by superimposing the infinitesimal perturbations $\mathbf{v}, \mathbf{b}$ and $p$ to the base flow variables $\mathbf{V}$, $\mathbf{B}$ and $P$. Then, the classical normal modes approach are used to linearize the resulting equations as follows.

$$
\begin{aligned}
\left(v_{x}, v_{y}, v_{z}\right) & =\left[\psi_{x}(y), \psi_{y}(y), \psi_{z}(y)\right] e^{i(\alpha x+\beta z-\alpha c t)}(8) \\
\left(b_{x}, b_{y}, b_{z}\right) & \left.=\left[\phi_{y}(y), \phi_{z}(y), \phi_{z}(y)\right)\right] e^{i(\alpha x+\beta z-\alpha c t)}(9) \\
p & =\delta(y) e^{i(\alpha x+\beta z-\alpha c t)}
\end{aligned}
$$

where $\psi(y), \phi(y)$ and $\delta(y)$ are, respectively, the complex amplitudes of the perturbations $\mathbf{v}, \mathbf{b}$ and $p$. Thus, $\alpha$ is the wave number, $c$ is the complex wave speed and $i^{2}=-1$. Using the Squire's theorem [10] by taking $\beta=0$. Then, by applying the Lock's assumption [6] for the highly electrically conducting fluid, $R e_{m}<<1$, the differential equation governing the fluid motion with the correspond boundary conditions are reduced to

$$
\begin{gathered}
\left(D^{2}-\alpha^{2}\right)^{2} \psi \\
-i \alpha \operatorname{Re}\left[\left(V_{b_{x}}-c\right)\left(D^{2}-\alpha^{2}\right)-D^{2} V_{b_{x}}\right] \psi \\
=\left[M^{2} D^{2}-R_{C}\left(D^{3}-\alpha^{2} D\right)\right] \psi \\
\psi(y= \pm 1)=D \psi(y= \pm 1)=0
\end{gathered}
$$

where $D$ represents differentiation with respect to $y$.

The equation(11) represents the classical OrrSommerfeld equation in which added two additional terms. The first term is due to the cross-flow $\left(R_{C}\right)$ and the second one, reflects the effect of an external magnetic field $(M)$. Next, the modified Orr-Sommerfeld equation(11) with the associated boundary conditions Eq.(12) are reduced to an algebraic system with $c$ $\left(=c_{r}+i c_{i}\right)$ eigenvalues: $\mathbf{E}\left(\alpha, R e, M, N, R_{C}\right) \cdot \psi=$ $c \mathbf{F}\left(\alpha,, R e, M, N, R_{C}\right) . \psi$.Then, the equations are solved numerically using the Chebyshev spectral collocation method with $N$ collocation points of Gauss-Lobatto. The accuracy of the numerical code has been checked through comparison with the results given in $[4,7,9]$. All numerical results presented in the present work are computed with $120 \mathrm{~N}$ collocation points.

\section{Results and conclusion}

Figures 1(a) and 1(b) exhibit the combined effect of a uniform cross-flow and an external transverse magnetic field on the dominant eigenmode, which correspond to the most unstable eigenmode, with its associated wave speed for $\alpha=1$ and $R e=6000$. It is shown in Fig.1(a) that, for $M=0$, the eigenmode initially is stabilizing ( $c_{\text {imax }}$ decreasing), then passes to a destabilization phase ( $c_{\text {imax }}$ increasing), and finally regains its stability for the large values of $R_{C}$. This result converges to that reported in [4]. 

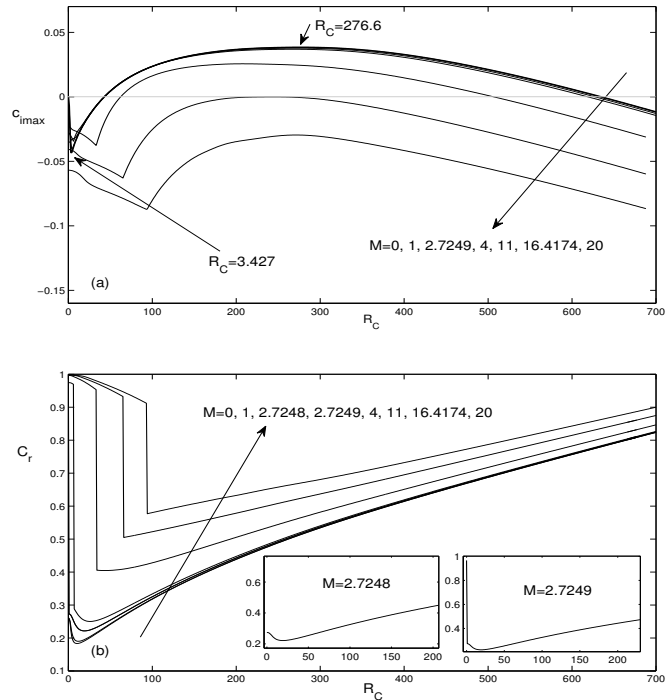

Fig.1 Combined effects of cross-flow Reynolds number, $R_{C}$ and Hartmann number, $M$ on: (a) Dominant eigenmode. (b) Wave speed associated to the dominant mode.

Holding the magnetic field strength no-zero, the largest value of $c_{\text {imax }}$, corresponding to the maximum of destabilization, decreases with an increase in Hartmann number which leads to stabilize the flow. The cross-flow Reynolds number, for which destabilization is maximized, decreases with the Hartmann number. The range of $R_{C}$ in which the cross-flow has a destabilizing effect narrows as $\mathrm{M}$ increases. These results suggest that the instability caused by cross-flow can be controlled by including an external magnetic field. Fig.1(b) shows that as $M$ is increased, the wave speed of the dominant eigenmode initially decreases and then increases with $R_{C}$. A jump phenomenon is observed when $M \geq 2.7249$. This jump is caused by a mode transition, which can be delayed by an increasing in $M$. Figures 1(a) with 1(b) suggest that, for large values of $R_{C}$, a mode transition is necessary to provoke the stabilizing character of the magnetic field.

For typical values of $R_{C}$ and $M$, figure (2) depicts the eigenfunction associated to dominant eigenmode. Fig.2(a) shown that, for the classical PPF ( $M=R_{C}=$ $0)$, the dominant eigenmode is the walls mode. In the case of PPF with cross-flow the eigenmode oscillations shift from the wall in which the fluid is injected towards the other one [see Figs. 2(a) and 2(d)]. Furthermore, in the presence of the magnetic field without cross-flow $R_{C}=0$, figures. 2 (a-c) confirm that, as $M$ is increased from 2.7248 to 2.7249 a mode transition is occurring from the wall mode to the central mode. Then it is extending symmetrically towards the both walls with $M$ as shown in Fig.2(f). The eigenfunctions in Figs. 2(e-g) clearly show that where $R_{C} \neq 0$ and $M \neq 0$ the both effects are superimposed.
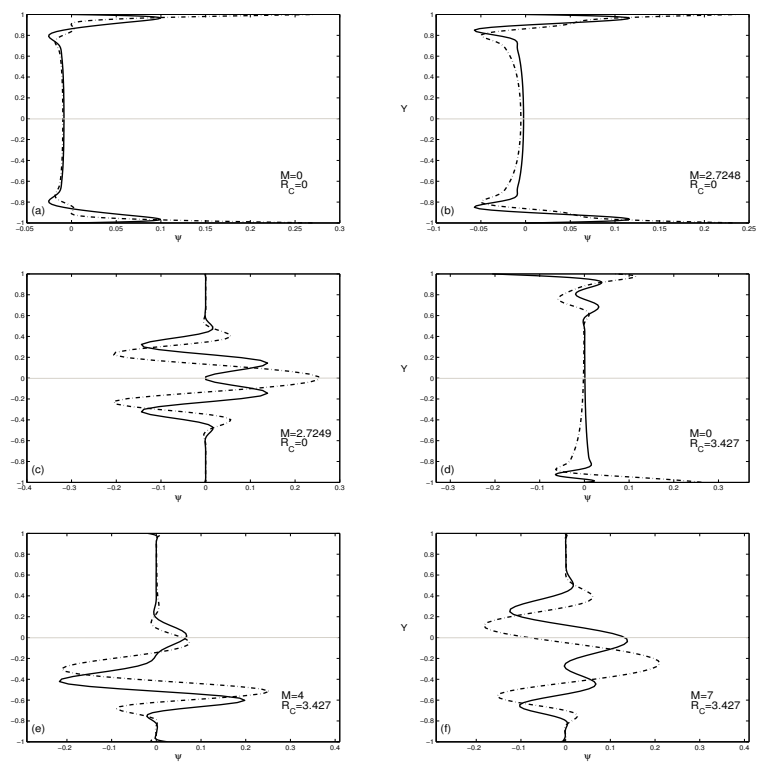

Fig.2 Eigenfunctions associated to the dominant modes as function of Hartmann number, $M$, and cross-flow Reynolds number, $R_{C}$.

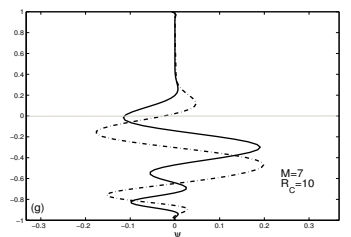

\section{References}

[1] A.S. Berman, Concerning laminar flow in channels with porous walls J. Appl. Phys. 27 (1956) 1557

[2] F. D. Hains, Stability of plan Couette-Poiseuille flow with uniform cross flow. Physics of Fluids, 14, 1620 (1971).

[3] D. M. Sheppard, Hydrodynamic stability of the flow between parallel porous walls Phys. Fluids. 15, 241 (1972).

[4] J. H. M. Fransson and P. H. Alfredsson, On the hydrodynamics stability of channel flow with cross flow Phys. Fluids. 15, 436 (2003).

[5] M. Lamine and A. Hifdi, stability analysis of plane couette-poiseuille flow with porous walls under transverse magnetic field, Journal of Thermal Engineering Vol. 2, No. 4, July, (2016), pp. 807-813.

[6] R. C. Lock, The stability of the flow of an electrically conducting fluid between parallel planes under a transverse magnetic field Proc. Roy. Soc. Lond. A. 221, 105 (1955).

[7] M. Takashima, Fluid Dynamics Research. 17, 293 (1996).

[8] K. N. Mehta and R. K. Jain, Laminar hydrodynamic flow in a rectangular channel with porous walls, Proc. Nat. Inst. Sci. India. 28, 846 (1962).

[9] A. Hifdi, M. Ouazzani Touhami and J. Khalid-Naciri, Channel entrance flow and its linear stability, J. Stat Mech.:Theor. Exp. P06003 (2004).

[10] H. B. Squire, On the stability of three dimensional disturbances of viscous flow between parallel walls Proc. R. Soc. Lond. 142, 621 (1933).

[11] R. K. Jain, Stability of hydromagnetic flow between porous parallel plates under transverse magnetic field, Nat. Inst. Sci. India Part A. 35, 37 (1969). 